\title{
Experimental investigation on destructive potential of shock-induced bubble collapse
}

\author{
${ }^{1}$ Zhaoguang Wang *; ${ }^{1}$ Thomas Hopfes; ${ }^{1}$ Marcus Giglmaier; ${ }^{1}$ Nikolaus Adams \\ ${ }^{1}$ Institute of Aerodynamics and Fluid Mechanics, Technical University of Munich, \\ Boltzmannstr. 15, D-85747 Garching, Germany
}

\begin{abstract}
The present study experimentally investigates shock-induced collapse of bubbles near boundaries of different plasticity and the resulting destructive erosion. Air bubbles held in gelatin are placed close to either a solid wall or a soft clay layer. High pressure impulse generated by a shock tube is employed to trigger bubble collapse. The bubble deformation is visualized by a focused shadowgraph system, and the damage caused to the boundary surface is closely examined as well. The collapse of bubbles near the soft clay layer shows differences from that near the solid wall in both qualitative description of bubble deformation and quantitative measurement of bubble dynamics. The surface of the clay is marked by the bubble collapse with a volcano-shaped imprint and some material floats into the gelatin in a mushroom shape. In this ongoing investigation, refined results under well-defined and uniform initial conditions will be provided and well suited for validation of numerical models and approaches.
\end{abstract}

Keywords: bubble collapse; cavitation erosion; shock wave

\section{Introduction}

The collapse of gas bubbles close to a boundary plays a key role in diverse research fields, such as the evaluation of cavitation damage to underwater propellers of ships [1] and the fragmentation of kidney stones in extracorporeal shock-wave lithotripsy (ESWL) [2, 3]. A deeper insight into the associated destructive mechanism helps to develop new techniques, especially in medical fields such as drug targeting and gene delivery [4]. Originating from the same physical phenomenon, topics with different emphases have been brought forward and investigated.

Bubble dynamics in the collapse near a solid boundary, which intends to analogize the surface of ship propellers or kidney stones, has been studied by a vast number of research. Benjamin \& Ellis [5] are among the pioneers introducing the aspherical collapse of near-wall bubbles and demonstrating the existence of a liquid jet induced towards the wall. The liquid jet piercers through the bubble and may further impinge on the wall $[6,7]$. Shima et al. [8] show the strength of the liquid jet is directly affected by the bubble-to-wall distance and decreases as the distance increases. Jones \& Edwards [9] and Naudé \& Ellis [10] first notice the emission of extra shock waves during bubble collapse. Ohl et al. [11] further explain such shock waves as results of jet impact upon the opposite side of the bubble and the collapse of the remaining toroidal bubble. The induced shock waves expand nearly spherically with the strength decaying and hit the wall normally $[8,12]$. The appearance of counter jets directed away from the boundary at the second collapse of bubbles is initially shown by Lauterborn \& Bolle [13]. Lindau \& Lauterborn [14] find the counter jet disappears if the laser-produced bubble gets in contact with the boundary when expanding to the maximum radius.

Another topic which deals with bubble collapse near soft materials gains increasing interests as to evaluate the damage to soft human tissues in ESWL. Gibson \& Blake [15] investigate collapse of a spark-generated bubble attached to the surface of vulcanized rubber and observe no liquid jet developed and the bubble splitting into separate parts. They conclude that the bubble behavior is notably influenced by the inertia and the stiffness of nearby boundaries. A laserinduced bubble collapsing near an elastic material which consists of a polyacrylamide mixed with water is studied by Brujan et al. [16]. Interesting features such as the formation of mushroom-shaped bubbles, an annular jet splitting the bubble and a pair of axial jets directed towards each other are observed. The work also shows important effects of the elastic modulus of the material and the stand-off distance on the bubble deformation. Similar results are obtained by Tomita \& Kodama [17] who employ a composite surface with either rigid or soft plates glued on a foam rubber and Shaw et al. [18] and Turangan et al. [19] who replace elastic boundaries with elastic membranes.

Apart from the bubble dynamics, study of the damage pattern caused by the bubble collapse on the nearby boundaries also draws attentions. Tomita \& Shima [20] and Philipp \& Lauterborn [21] investigate the effect of the stand-off distance on the boundary erosion and show similar circular pit shapes. Influence of the physical properties of boundaries is studied by Kodama \& Takayama [22] who conclude that even gelatin and rat livers share similar acoustic impedance, the discrepancy in their elasticity results in different damage patterns. A few researchers [23, 24] also 
show that another factor determining the erosion is the way to generate bubbles, such as acoustic and hydrodynamic means, electric discharge and optical breakdown.

Although extensive research has been conducted concerning bubble collapse as shown above, to the authors' best knowledge, it still remains a question to answer: what is the dominate destructive mechanism. Impingement of the liquid jet, collapse of the main bubble and collapse of the tiny bubble clouds induced by the liquid jet all contribute to the cavitation damage to certain extent, but no agreement has been achieved on the relative significance of these factors. New features in the bubble dynamics when a soft boundary appears nearby add even more complexity to this problem. Motivated to cast slightly more light on understandings of the underlying physics, the current work studies the shock-induced collapse of air bubbles in gelatin close to different boundaries, namely a solid wall and a soft clay layer. The radius change and the centroid movement of bubbles in two cases are compared. Furthermore, surface damage caused by nearby bubble collapse is analyzed.

\section{Experimental setup}

To acquire an impulsive high pressure for bubble collapse, experiments are carried out in a shock tube with an inner diameter of $290 \mathrm{~mm}$. Figure 1 depicts the layout of the shock tube and the corresponding measurement system. The $3 \mathrm{~m}$ long driver section and the $19.5 \mathrm{~m}$ long driven section are separated by a metal diaphragm which is placed against a downstream X-shaped sharp blade. A $0.5 \mathrm{~m}$ long test section, of which the cross section is transformed by a cookiecutter to a $190 \mathrm{~mm} \times 190 \mathrm{~mm}$ square, is attached to the end of the driven section. In the experiments, air is pumped into the driver section continuously. When the critical pressure ( 8.0 bar for the current setup) is reached, the diaphragm breaks and a shock wave develops rapidly propagating towards the test section.

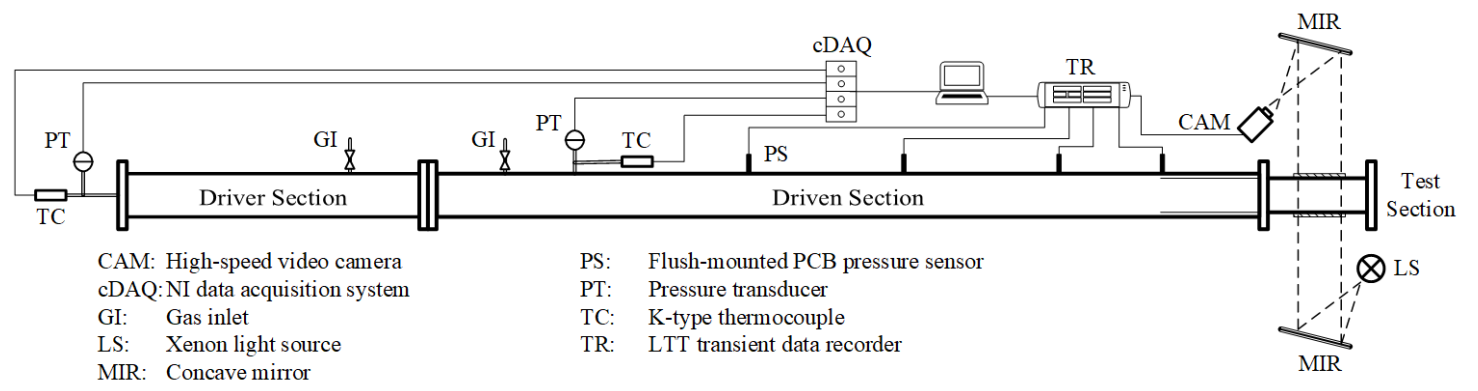

Figure 1: Experimental arrangement of the shock tube for bubble dynamics experiments

Initial experimental pressure and temperature conditions are measured by pressure transducers and K-type thermocouples, respectively. The output signals are acquired by a NI ${ }^{\circledR} \mathrm{cDAQ}$ device at a sampling rate of $1 \mathrm{kHz}$. A few meters upstream of the test section, four PCB Piezotronics ICP ${ }^{\circledR}$ fast-response pressure sensors are flush-mounted on the shock tube, monitoring pressure changes inside the tube during the experiments. The $4 \mathrm{MHz}$ transient pressure data are recorded by a $\mathrm{LTT}^{\circledast}$ data acquisition device.

A highly sensitive Z-type schlieren system is built to visualize the flow field. A $150 \mathrm{~W}$ xenon lamp serves as the light source and a Shimazu ${ }^{\circledR}$ Hyper Vision HPV-X ultra-high-speed camera $(250 \times 400$ pixels with a frame rate of up to $5 \mathrm{MHz}$ ) records 128 continuous frames of the bubble deformation. For the present results, the knife edge in the optical system is removed to guarantee adequate brightness of images captured. Therefore, the original schlieren system is simplified into a focused shadowgraph.

The sketch in fig. 2 describes a new experimental test section that will be shown at the conference. However, due to delays in the manufacturing process, a workaround with an acrylic box placed inside an already existing test chamber is applied to obtain preliminary results. Deformation of the acrylic box and extra wave motion in the surrounding air of the box bring undesired effects. But main features of the bubble collapse are well captured.

In terms of the new test section, the downstream $400 \mathrm{~mm}$-long part is filled with gelatin, which is made from a mixture of distilled water, Gelrite ${ }^{\circledR}$ Gellen gum and magnesium sulfate with the mass ratio of $10000: 6: 5$. The rear wall is either exposed to serve as a solid boundary or covered with a $4 \mathrm{~mm}$-thick layer of soft clay. An air bubble is produced carefully with a syringe close to the boundary surface under atmospheric conditions ( $p_{0} \approx 1 \mathrm{bar}$ ). The solidity and the 
viscosity of the water-like gelatin contribute to hold the air bubble at a fix position although increasing the difficulty in producing a perfectly spherical bubble. One thing to notice is that the gelatin is a non-Newtonian fluid but behaves like water under high stresses.

The $x$ - $t$ diagram in fig. 2 illustrates the wave motion inside the test section and the temporal pressure distribution involved. When the incident shock (S) generated by the shock tube arrives at the air-gelatin interface, it is reflected back almost ideally (RS) due to the large acoustic impedance difference between air and the gelatin. Based on the pressure balance at the air-gelatin interface, a compression wave $(\mathbf{C})$ travels through the gelatin at the sound speed of around $1500 \mathrm{~m} / \mathrm{s}$. Since the bubble-to-wall distance is small, the pressure field surrounding the bubble is raised up to a considerably high value by the upcoming compression wave (C) and the subsequent reflected compression wave (RC) in a negligibly short time. The far-field pressure for the bubble collapse remains constant until an expansion wave $(\mathbf{E})$, which originates from the interaction of the reflected compression wave (RC) with the interface, arrives at the air bubble. For the example shown here, a gelatin filled test chamber with a length of $\ell=400 \mathrm{~mm}$ gives a test window of $\Delta t=0.5 \mathrm{~ms}$, which is sufficient for the primary (and secondary) collapse of a bubble with a diameter of $d=10 \mathrm{~mm}$ under a surrounding pressure of $p=10 \mathrm{bar}$ as estimated by the Rayleigh-Plesset equation.

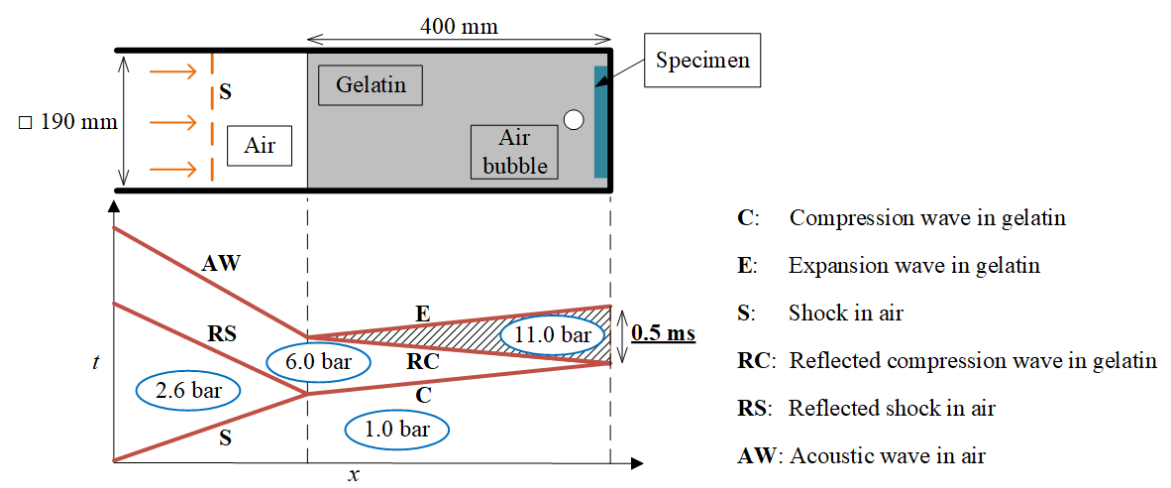

Figure 2: Sketch of the new test section with a wave motion diagram ( 8.0 bar in the driver section and 1.0 bar in the driven section)

\section{Preliminary results and discussions}

As aforementioned, the present work was carried out with the workaround setup which accounts for major disturbances in shadowgraph images displayed below. Also the observations to be discussed are confined to the current specific cases. The uncertainties and the repeatability will be evaluated with more upcoming experiments using the new test chamber as described in fig. 2 .

Air bubble collapse close to two different types of boundaries is studied. One is a solid wall, and the other is a $4 \mathrm{~mm}$ thick soft clay layer. In both experiments, the relative stand-off distance $\gamma=s / R_{0}(s$ : the distance from the bubble center to the boundary surface, $R_{0}$ : the initial bubble radius) is similar. Therefore, it is possible to make quantitative comparisons between these cases. For all following images, considering the asymmetric deformation of bubbles, the radius of an equivalent spherical bubble consisting of the same amount of dark pixels is calculated to represent $R$.

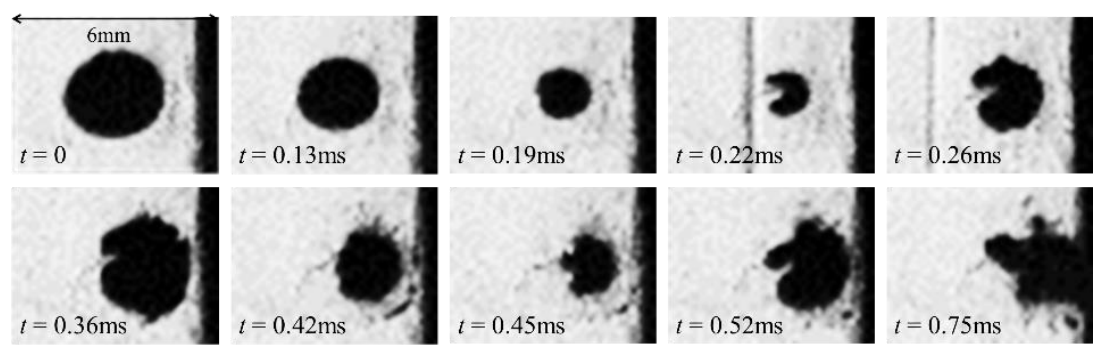

Figure 3: Bubble deformation close to the solid wall $\left(R_{0}=2.87 \mathrm{~mm}, \gamma=1.75\right)$ 
Figure 3 shows the bubble deformation close to the solid wall with $R_{0}=2.87 \mathrm{~mm}$ and $\gamma=1.75$. Images are recorded by the focused shadowgraph system at a sampling rate of 100000 frames/s. The bubble starts to shrink after the surrounding pressure is raised instantaneously by shock waves. At time $t=0.19 \mathrm{~ms}$, the off-wall side of the bubble has moved through a considerably longer distance than the near-wall side, and becomes flat or even slightly concave at middle. At $t=0.22 \mathrm{~ms}$, the bubble collapses to the minimum volume. The curved off-wall surface of the bubble clearly indicates the development of a liquid jet. The straight dark lines in this and the next images are shock waves in the surrounding air of the acrylic box. At $t=0.36 \mathrm{~ms}$, the re-expansion ends and the bubble recovers to nearly the initial size. The small-scale irregularities at the bubble surface facing towards the boundary suggest the liquid jet has impacted on it. Due to the aspherical bubble shape at the beginning of the second collapse, the bubble deforms into an apple shape at $t=0.45 \mathrm{~ms}$. After more succeeding expansions and compressions, the bubble lands on the boundary surface at $t=0.75 \mathrm{~ms}$. A crowd of smaller bubbles, which are shed off from the main bubble, are identified.

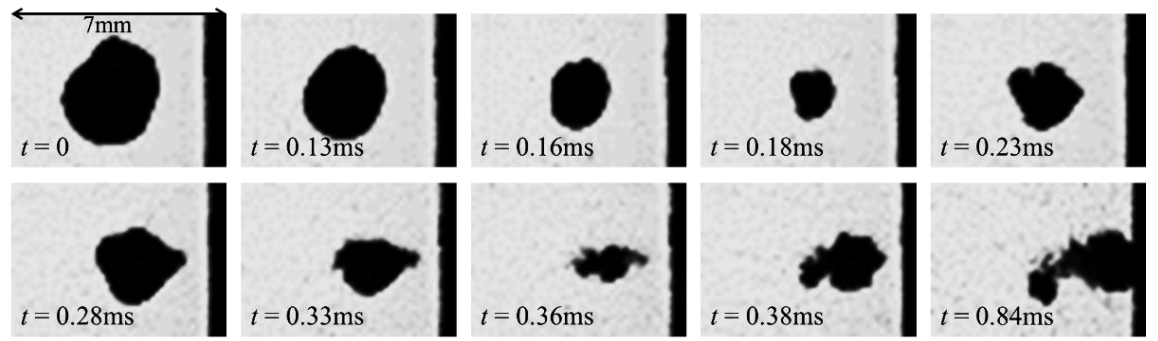

Figure 4: Bubble deformation close to the soft clay layer $\left(R_{0}=2.95 \mathrm{~mm}, \gamma=1.85\right)$

Figure 4 shows the bubble deformation near the soft clay with $R_{0}=2.95 \mathrm{~mm}$ and $\gamma=1.85$. The interframe time of the full video is $\Delta t=0.01 \mathrm{~ms}$. At $t=0.16 \mathrm{~ms}$, a liquid jet starts to develop as the off-wall surface of the bubble turns flat. The minimum volume of the first collapse is reached $0.02 \mathrm{~ms}$ later. The bubble finishes the re-expansion at $t=0.28 \mathrm{~ms}$ and a "nose" extrudes out of the bubble suggesting the penetration of the liquid jet. Then the funnel-shaped bubble begins to collapse again and tends to split into two parts at $t=0.36 \mathrm{~ms}$. The larger part expands further downstream and finally arrives at the boundary at $t=0.84 \mathrm{~ms}$, while the center of the smaller part floats towards the opposite direction slightly.

Although liquid jets appear in both cases, bubbles in fig. 3 and fig. 4 exhibit quite differently as getting closer to the boundary in the second collapse and the following oscillations. Moreover, no shock wave emission is observed during either bubble collapse. The low trigger pressure $p_{\infty}$ around the bubbles $\left(p_{\infty} / p_{0} \sim 10\right)$ and the insufficient framing rate (100000 frames/s) are responsible for this.

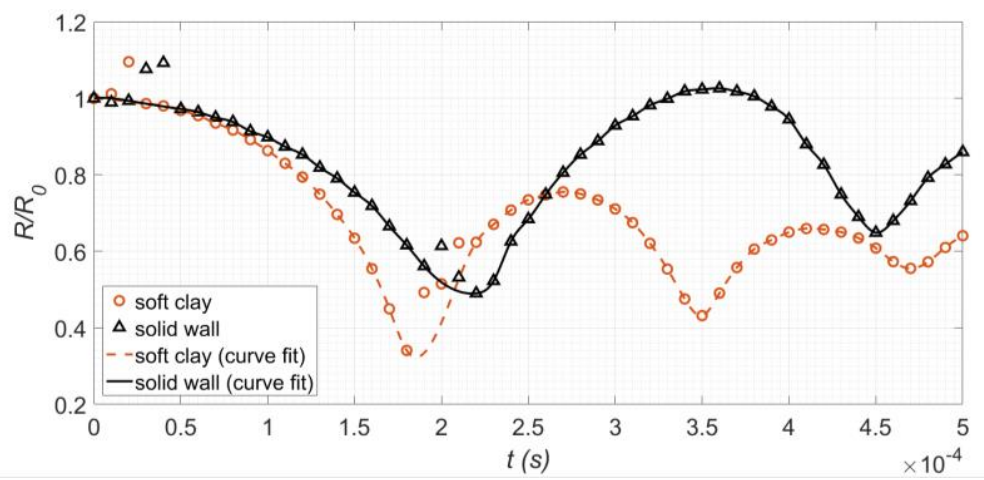

Figure 5: Comparison of the bubble radius changes between two cases (for the soft clay $t_{\mathrm{c}}=0.18 \mathrm{~ms}, R_{\min } / R_{0}=0.34$; for the solid wall $t_{\mathrm{c}}=0.22 \mathrm{~ms}, R_{\min } / R_{0}=0.49$ )

The temporal bubble radius changes of the two cases are compared in fig. 5, where $R$ is normalized against the initial bubble radius $R_{0}$. Both curves in the figure experience oscillations with the attenuation of amplitude. It is noticed that the bubble close to the solid wall re-expands to a radius slightly higher than the initial radius (for the dark solid line, $R / R_{0}>1$ at $t=0.35 \mathrm{~ms}$ ). Such an unexpected behavior could result from the irregularity of the bubble shape and the lack of information about internal bubble geometries from the two-dimensional images. Nevertheless, clear differences can still be distinguished between two cases. The bubble close to the soft clay collapses faster (collapse 
time $\left.t_{\mathrm{c}}=0.18 \mathrm{~ms}\right)$ than that near the solid wall $\left(t_{\mathrm{c}}=0.22 \mathrm{~ms}\right)$ (note that the former case even has a slightly larger initial bubble $R_{0}=2.95 \mathrm{~mm}$ vs $\left.2.87 \mathrm{~mm}\right)$, achieves a lower minimum normalized radius $\left(R_{\min } / R_{0}=0.34 v s 0.49\right)$ and has a stronger decay of the bubble oscillation. This could be explained by the previous knowledge that the pressure around the bubble is reduced more with the presence of a nearby solid boundary than a soft one.

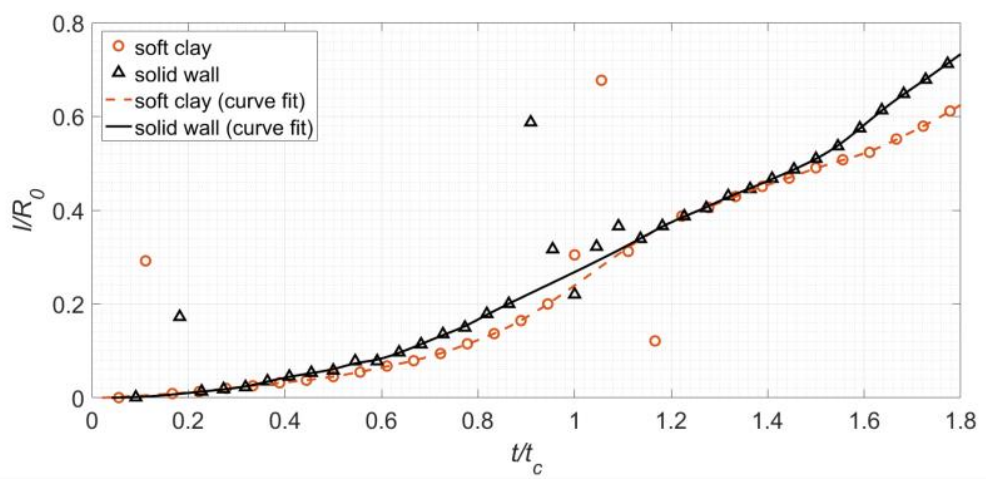

Figure 6: Comparison of the bubble centroid movement between two cases (within the first collapse $t / t_{\mathrm{c}}<1, l / R_{0}$ for the solid wall is higher than that for the soft clay, representing a stronger attraction for the bubble)

Comparison of bubble centroid movement is made between two cases in fig. 6, where the bubble centroid is determined as the area-weighted position of the dark pixels constituting the bubbles. The time $t$ and the displacement $l$ of the bubble centroids relative to the initial location are normalized by the corresponding collapse time $t_{\mathrm{c}}$ and the initial bubble radius $R_{0}$, respectively. Both bubbles travel to the boundary continuously but at different paces. During the first collapse $\left(t / t_{\mathrm{c}}<1\right)$, the bubble close to the solid wall shifts more, while both bubbles share overlapped locations during the re-expansion period (roughly with $t / t_{\mathrm{c}}$ from 1 to 1.55). Overall speaking, because of the lack of deformability, the solid wall attracts nearby bubbles more.
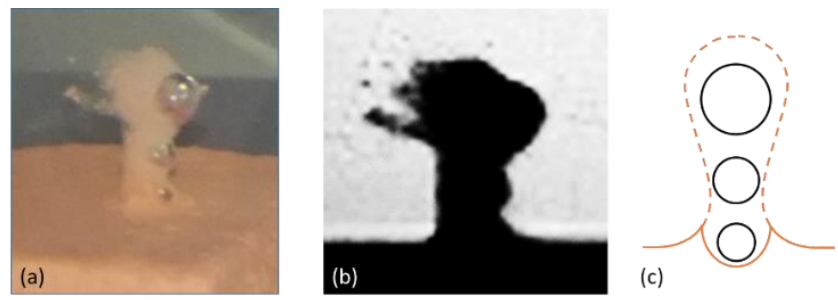

Figure 7: Surface damage of the soft clay layer caused by the bubble collapse: (a) a full-color image, (b) a shadowgraph image and (c) a simplified sketch

Apart from the bubble dynamics, the damage caused by the bubble collapse on the boundary is another concern. In terms of the solid wall, no visible imprint of damage is observed. As for the soft clay layer, however, distinct deformation is resulted. As shown in fig. 7, the bubble finally splits into three parts, with the smallest one inserted into the soft clay. Suffered from the crash of the bubbles, the surface of the boundary deforms into a volcano-shaped dimple with a mushroom-shaped clay cloud distributing around. This is a clear indication of the destructive potential of bubble collapse, which would have a significant impact on drug targeting and gene delivery. Due to the lack of physical properties of boundaries, further quantitative analysis is not given here.

\section{Conclusion and outlooks}

Air bubble collapse close to different boundaries, namely a solid wall and a soft clay layer, was studied experimentally. Major difference in the pattern of the bubble deformation was found in the second collapse, where the bubble next to the solid wall deformed into an apple shape, while in the other case the bubble split into two smaller parts. Quantitative analysis of the bubble dynamics further indicated that the bubble near the soft clay collapsed faster to a lower minimum radius and showed a stronger reduction in the amplitude of subsequent oscillations. The overall trend of the bubble centroid movement was similar in both experiments, except that the bubble moved faster towards the boundary when the solid wall was employed. In addition, the surface of the soft clay was left with a volcano-shaped dimple by the bubble collapse and some mass fell off forming a mushroom-shaped cloud surrounding the bubbles. Note that all the 
observations are only based on the present particular cases. More experiments will be conducted to quantify uncertainties and to estimate the repeatability before asserting any conclusion.

Since the current preliminary experimental rig was not built intentionally to investigate bubble dynamics, some important information about the experiments, like the pressure change around the bubble, is not obtainable. A new test chamber especially planned for these experiments is in preparation (as described in fig. 2), which has a stronger structure to allow for a higher operating pressure, larger glass windows with higher optical qualities to visualize the flow field better, and a more flexible arrangement to control positions of bubbles more accurately and to render a wider variety of boundaries possible. Therefore, more highly-resolved outcomes are expected in the near future and will be shown on the conference. Considering the new setup is capable to provide well-defined and uniform initial conditions for the bubble collapse, the upcoming refined results could serve to validate numerical models and approaches.

\section{References}

[1] Knapp, R. T. (1955). Recent investigations of the mechanics of cavitation and cavitation damage. Transactions of the ASME, 77, $1045-1054$.

[2] Crum, L. A. (1988). Cavitation microjets as a contributory mechanism for renal calculi disintegration in ESWL. The Journal of urology, 140(6), 1587-1590.

[3] Delius, M., Brendel, W., \& Heine, G. (1988). A mechanism of gallstone destruction by extracorporeal shock waves. Naturwissenschaften, 75(4), 200-201.

[4] Unger, E. C., Porter, T., Culp, W., Labell, R., Matsunaga, T., \& Zutshi, R. (2004). Therapeutic applications of lipid-coated microbubbles. Advanced drug delivery reviews, 56(9), 1291-1314.

[5] Benjamin, T. B., \& Ellis, A. T. (1966). The collapse of cavitation bubbles and the pressures thereby produced against solid boundaries. Philosophical Transactions of the Royal Society of London A: Mathematical, Physical and Engineering Sciences, 260(1110), 221-240.

[6] Brujan, E. A., Keen, G. S., Vogel, A., \& Blake, J. R. (2002). The final stage of the collapse of a cavitation bubble close to a rigid boundary. Physics of Fluids, 14(1), 85-92.

[7] Klaseboer, E., Hung, K. C., Wang, C., Wang, C. W., Khoo, B. C., Boyce, P., ... \& Charlier, H. (2005). Experimental and numerical investigation of the dynamics of an underwater explosion bubble near a resilient/rigid structure. Journal of Fluid Mechanics, 537, 387-413.

[8] Shima, A., Takayama, K., Tomita, Y., \& Miura, N. (1981). An experimental study on effects of a solid wall on the motion of bubbles and shock waves in bubble collapse. Acta Acustica united with Acustica, 48(5), 293-301.

[9] Jones, I. R., \& Edwards, D. H. (1960). An experimental study of the forces generated by the collapse of transient cavities in water. Journal of Fluid Mechanics, 7(4), 596-609.

[10] Naudé, C. F., \& Ellis, A. T. (1961). On the mechanism of cavitation damage by nonhemispherical cavities collapsing in contact with a solid boundary. Journal of Basic Engineering, 83(4), 648-656.

[11] Ohl, C. D., Philipp, A., \& Lauterborn, W. (1995). Cavitation bubble collapse studied at 20 million frames per second. Annalen der Physik, 507(1), 26-34.

[12] Klaseboer, E., Fong, S. W., Turangan, C. K., Khoo, B. C., Szeri, A. J., Calvisi, M. L., ... \& Zhong, P. (2007). Interaction of lithotripter shockwaves with single inertial cavitation bubbles. Journal of fluid mechanics, 593, 33-56.

[13] Lauterborn, W., \& Bolle, H. (1975). Experimental investigations of cavitation-bubble collapse in the neighbourhood of a solid boundary. Journal of Fluid Mechanics, 72(2), 391-399.

[14] Lindau, O., \& Lauterborn, W. (2003). Cinematographic observation of the collapse and rebound of a laser-produced cavitation bubble near a wall. Journal of Fluid Mechanics, 479, 327-348.

[15] Gibson, D. C., \& Blake, J. R. (1982). The growth and collapse of bubbles near deformable surfaces. Applied Scientific Research, 38(1), 215224.

[16] Brujan, E. A., Nahen, K., Schmidt, P., \& Vogel, A. (2001). Dynamics of laser-induced cavitation bubbles near an elastic boundary. Journal of Fluid Mechanics, 433, 251-281.

[17] Tomita, Y., \& Kodama, T. (2003). Interaction of laser-induced cavitation bubbles with composite surfaces. Journal of applied physics, 94(5), 2809-2816.

[18] Shaw, S. J., Jin, Y. H., Gentry, T. P., \& Emmony, D. C. (1999). Experimental observations of the interaction of a laser generated cavitation bubble with a flexible membrane. Physics of Fluids, 11(8), 2437-2439.

[19] Turangan, C. K., Ong, G. P., Klaseboer, E., \& Khoo, B. C. (2006). Experimental and numerical study of transient bubble-elastic membrane interaction. Journal of Applied Physics, 100(5), 054910.

[20] Tomita, Y., \& Shima, A. (1986). Mechanisms of impulsive pressure generation and damage pit formation by bubble collapse. Journal of Fluid Mechanics, 169, 535-564.

[21] Philipp, A., \& Lauterborn, W. (1997). Damage of solid surfaces by single laser-produced cavitation bubbles. Acta Acustica united with Acustica, 83(2), 223-227.

[22] Kodama, T., \& Takayama, K. (1998). Dynamic behavior of bubbles during extracorporeal shock-wave lithotripsy. Ultrasound in medicine \& biology, 24(5), 723-738.

[23] Dular, M., Delgosha, O. C., \& Petkovšek, M. (2013). Observations of cavitation erosion pit formation. Ultrasonics sonochemistry, 20(4), 1113-1120.

[24] Tzanakis, I., Eskin, D. G., Georgoulas, A., \& Fytanidis, D. K. (2014). Incubation pit analysis and calculation of the hydrodynamic impact pressure from the implosion of an acoustic cavitation bubble. Ultrasonics sonochemistry, 21(2), 866-878. 